\title{
A novel double-stranded RNA mycovirus isolated from Trichoderma harzianum
}

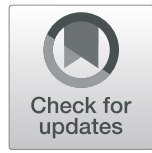

\author{
Chenchen Liư ${ }^{\dagger}$, Mei Li ${ }^{\dagger}$, Estifanos Tsegaye Redda, Jie Mei, Jiantai Zhang, Beilei Wu ${ }^{*}$ and Xiliang Jiang ${ }^{*}$
}

\begin{abstract}
Background: Trichoderma spp. are used extensively in agriculture as biological control agents to prevent soil-borne plant diseases. In recent years, mycoviruses from fungi have attracted increasing attention due to their effects on their hosts, but Trichoderma mycoviruses have not been the subject of extensive study. We sought to discover novel mycoviruses from Trichoderma spp. and to determine the effects of the biocontrol function of Trichoderma spp.

Methods: Mycoviruses were screened by dsRNA extraction and metagenomic analysis. RT-PCR, 5' RACE, and 3' RACE were used to obtain the genome sequence. MEGA software was used to classify the new mycovirus. The effects of the identified mycovirus on the biological properties of the host strain 525 were evaluated using cucumber plants and Fusarium oxysporum f. sp. cucumerinum.
\end{abstract}

Results: A novel mycovirus, Trichoderma harzianum mycovirus 1 (ThMV1) (accession number MH155602), was discovered in Trichoderma harzianum strain 525, a soil-borne fungus collected from Inner Mongolia, China. The mycovirus exhibited a double-stranded RNA (dsRNA) genome with a complete genome sequence of 3160 base pairs and two open reading frames (ORFs) on the negative strand. Phylogenetic analysis indicated that it belongs to an unclassified family of dsRNA mycoviruses. The removal of ThMV1 from the host 525 strain reduced host biomass production and improved the biocontrol capability of the host for Fusarium oxysporum f. sp. cucumerinum. At same time, the presence of ThMV1 improved the growth of cucumber.

Conclusion: ThMV1 is a new unclassified mycovirus found in T. harzianum. It not only affects the phenotype of the host strain but also reduces its biocontrol function, which sheds light on the interaction between the mycovirus and Trichoderma spp.

Keywords: Mycovirus, Trichoderma harzianum, dsRNA, Trichoderma harzianum mycovirus 1

\section{Background}

Mycoviruses are widespread viruses that infect filamentous fungi and yeasts, and most do not cause their hosts to exhibit obvious symptoms $[1,2]$. The majority of mycoviruses exhibit a double-stranded RNA (dsRNA) genome, which is diagnostic. However, a few mycoviruses exhibit single-stranded RNA (ssRNA), doublestranded DNA (dsDNA), or single-stranded DNA (ssDNA) genomes [3]. Based on the mode of replication and the type of genome, the International Committee on Taxonomy of Viruses has divided all currently known mycoviruses into 16 families and an unclassified group.

\footnotetext{
* Correspondence: beileiwu@foxmail.com; jiangxiliang@caas.cn

${ }^{+}$Chenchen Liu and Mei Li contributed equally as first authors

Institute of Plant Protection, Chinese Academy of Agricultural Sciences, No.2 West Yuanmingyuan Rd., Haidian District, Beijing 100193, People's Republic of China
}

(c) The Author(s). 2019 Open Access This article is distributed under the terms of the Creative Commons Attribution 4.0 International License (http://creativecommons.org/licenses/by/4.0/), which permits unrestricted use, distribution, and reproduction in any medium, provided you give appropriate credit to the original author(s) and the source, provide a link to the Creative Commons license, and indicate if changes were made. The Creative Commons Public Domain Dedication waiver (http://creativecommons.org/publicdomain/zero/1.0/) applies to the data made available in this article, unless otherwise stated. positive-sense ssRNA virus families, two reverse-transcription virus families (+ssRNA), one negative-sense ssRNA virus family, and one positive-sense ssDNA virus family [4]. The taxonomic status of approximately $20 \%$ of fungal viruses has yet to be determined $[5,6]$.

Mycoviruses are transferred from one fungal isolate to another through hyphal fusion (anastomosis) or from parent to offspring via spores (mostly via fusion of asexual spores but also sexual spores). Some mycoviruses can be eliminated in sexual spores. For example, ssRNA and dsRNA viruses in Basidiomycetes can be transmitted by basidiospores, but they can also be removed from the basidiospores produced by Helicobasidium mompa [7]. In some cases, they can be virulent to their hosts, but most mycovirus infections are asymptomatic [8]. 
In recent years, mycoviruses have drawn increased attention for the effects they can have on their host virulence [9]; some can significantly reduce virulence (e.g., the dsRNA virus DK21 of Fusarium graminearum [10]), while others can significantly increase it (e.g., the dsRNA mycovirus RsRV2 found in the rice sheath blight isolate D122 strain [11]). These findings have spurred the development of hypovirulence-associated mycoviruses for use as biocontrol agents [12-14].

Mycoviruses exhibit two main mechanisms that facilitate their function as biocontrol agents of plant pathogenic fungi: first, they can cause the host to become a low-virulence strain; second, the metabolites induced by the mycovirus can increase the pathogenicity of the host [15-17]. Although some low-virulence mycoviruses have been found in phytopathogenic fungi, most are still in the research stage examining their potential as biocontrol agents. The most successful mycovirus biocontrol agent identified to date has been Cryphonectria parasitica hypovirus 1 (CHV1), which was the first low-virulence mycovirus employed to both prevent and treat disease [18].

Trichoderma spp. are anamorphic soil-borne filamentous fungi classified as belonging to phylum Ascomycota, class Sordariomycetes, order Hypocreales, and family Hypocreales. While they are well studied [19], their mycoviruses have not been sufficiently investigated [20].

Here, we chose 150 strains of Trichoderma spp. isolated from Xinjiang, Inner Mongolia, Jilin, and Heilongjiang Provinces, China, to screen mycoviruses using metagenomics and molecular biology methods. One mycovirus was recovered, and its genome and molecular characteristics are described along with its biological properties.

\section{Methods}

\section{Fungal strains}

One hundred and fifty Trichoderma spp. strains were isolated from the forests and grasslands of Xinjiang, Inner Mongolia, Jilin, and Heilongjiang Provinces, China, in 2014-2016 (Additional file 1: Table S1). All fungal strains were cultured on potato dextrose agar (PDA) plates for 7 days at $28^{\circ} \mathrm{C}$.

\section{Extraction and purification of dsRNA}

dsRNA was extracted from the mycelia using CF-11 cellulose column chromatography [21]. To isolate dsRNA, the mycelia were grown for 7-10 days on cellophane membranes placed on top of the PDA medium in Petri dishes. Following harvest, the mycelia were stored at $80^{\circ} \mathrm{C}$. dsRNA was extracted according to a previously described method [21] and then treated with RNase-free DNase I (TaKaRa, Dalian, China) and S1 Nuclease (TaKaRa, Dalian, China) following the manufacturer's instructions to remove any DNA and ssRNA contamination. Finally, electrophoresis in a $1.5 \%$ agarose gel was performed, and $\mathrm{t}$ the dsRNA was detected by UV transillumination.

\section{Metagenomic sequencing}

dsRNA was extracted and sequenced using high-throughput sequencing (Illumina HiSeq 2000/2500) at the Biotechnology Corporation (Biotechnology Corporation, Shanghai, China). Briefly, purified dsRNA was first assessed for quality. Ribosomal RNA (rRNA) was removed, and the dsRNA was fragmented. Next, first-strand complementary DNAs (cDNAs) were synthesized, followed by second-strand cDNAs. The complete sample sequencing library was obtained, and the full library was sequenced. The sequencing data were then analysed; unigenes were annotated with RefSeq; and non-redundant proteins according to the National Center for Biotechnology Information (NCBI) Basic Local Alignment Search Tool (BLAST) were searched to screen mycovirus-related sequences.

\section{Cloning and sequencing of the mycovirus}

cDNA of the dsRNA were obtained by reverse-transcription polymerase chain reaction (RT-PCR) based on the contig obtained from metagenomics analysis (forward primer: 5' TCGATGTACGGATTCTCGTGC 3'; reverse primer: 5' TGTCATCATCGTCTTCAGCCC 3'; fragment length: $2997 \mathrm{bp}$ ). Having obtained most of the virus sequence, the $5^{\prime}$ - and the $3^{\prime}$-termini were cloned using the rapid amplification of cDNA ends (RACE) method. The $5^{\prime}$-end was obtained by the classic $5^{\prime}$-RACE cloning method [22] (Additional file 1: Table S3). A poly(A) tail was added to the 3 '-end of the cDNA using terminal deoxynucleotidyl transferase (TDT). Linker primers and specific primers were used to perform nested PCR amplification. The 3 '-end of the virus was obtained through the 3'-RACE cloning method [23] (Additional file 1: Table S3). Using the QT hybrid primer software, specific primers were designed for nested PCR amplification.

The RT-PCR products were purified using a gel extraction kit (Tiangen, Germany), ligated to the PMD18-T vector, and submitted for sequencing. Using DNAMAN software, the cDNA clone sequence of the virus and the RACE clone sequence were assembled to form a complete viral genome sequence.

\section{Complete genome sequence and phylogenetic analysis} Mycovirus ORFs were identified by the ORF finder program from the NCBI website (https://www.ncbi.nlm.nih. gov/orffinder/). The mycovirus sequence was searched using the NCBI BLAST program to identify similar mycoviruses for phylogenetic analysis. The phylogenetic tree was constructed using the maximum likelihood method for the 29 chosen mycovirus sequences of RdRP, 22 mycovirus sequences of hypothetical proteins or CPs, and $22 \mathrm{RdRP}+$ hypothetical proteins or CPs using MEGA 
6.0 software based on the best models of nucleotide substitution: $\mathrm{K} 2+\mathrm{G}+\mathrm{I}, \mathrm{LG}+\mathrm{G}+\mathrm{I}+\mathrm{F}$, and $\mathrm{rtREV}+\mathrm{G}+\mathrm{I}+\mathrm{F}$ (Additional file 1: Tables S4, S5 and S6).

\section{Effect of the mycovirus on the biological properties of Trichoderma strain $\mathbf{5 2 5}$}

Tthe inhibitory function of ribavirin was used to establish an analysis system for eliminating mycovirus from Trichoderma strain 525 by protoplasting/regeneration. The enzymatic hydrolysate used for the preparation of protoplasts of Trichoderma strain 525 was Trichoderma lysing enzyme (BingDa Biotechnology Company, Beijing). After enzymatic lysis, a $200 \mu \mathrm{L}$ aliquot of protoplasts was applied to regeneration medium that contained ribavirin $(100 \mu \mathrm{M})(\mathrm{BingDa}$ Biotechnology Company, Beijing), followed by culture at $28^{\circ} \mathrm{C}$ for 2 days. Single colonies were then picked and transferred to PDA medium that contained ribavirin at $100 \mu \mathrm{M}$, followed by culture for 7 days. To check if the mycovirus was present, dsRNA was extracted [24], after which RT-PCR and northern blotting were used to reconfirm whether dsRNA had been eliminated from the Trichoderma strain. A digoxigenin (DIG)-labelled probe was synthetized using the PCR DIG Probe Synthesis Kit (Roche Diagnostics, USA) with the following RT-PCR primers: forward primer: 5' TCGATGTACGGATTCTCGTGC 3'; reverse primer: 5' TGTCATCATCGTCTTCAGCCC 3'. The length of the RT-PCR fragment was $2997 \mathrm{bp}$.

The biological functions of the 525 strain with the mycovirus (T525) and the strain without the mycovirus (T525-F) were evaluated. Both strains were cultured in PDA, corn meal dextrose agar (CMD) medium, and Czapek-Dox (CZA) medium at $28^{\circ} \mathrm{C}$ for 8 days. The length of hyphal growth was measured every $24 \mathrm{~h}$, with thirty replicates in each case. To obtain the differences between the strains, the hyphal growth rates of the two strains were compared with each other. The T525 and T525-F strains were inoculated into PD liquid medium and incubated at $28^{\circ} \mathrm{C}$ with $180 \mathrm{rpm}$ shaking for 2 days. The dry weights of the mycelia in each bottle were then determined, and the biomasses of the two strains were compared, with twenty replicates in each case.

To evaluate biocontrol capabilities in vitro, antagonistic experiments involving T525 and T525-F were carried out. The three pathogens Fusarium oxysporum f.sp. cucumebrium Owen, Botrytis cinerea, and Fusarium oxysporum f. sp. vasinfectum were chosen for dual culture to analyse the characteristics of the antagonism of T525 and T525-F.

The biocontrol effect of T525 and T525-F against $F$. oxysporum f. sp. cucumerinum was further explored in vivo in cucumber plants. Six treatments were designed (Additional file 1: Figure S4): T1, in which cucumber seeds were planted in plates with sterilized water and incubated at $28^{\circ} \mathrm{C}$ until the 6th day, after which the seedlings were transferred to glass test tubes (GTTs) containing $10 \mathrm{~mL}$ of $1 / 8 \mathrm{MS} ; \mathrm{T} 2$, in which cucumber seeds were planted in plates with sterilized water and then incubated at $28^{\circ} \mathrm{C}$ until the 4th day, after which the seedlings were exposed with $1 \times 10^{7} \mathrm{CFU} / \mathrm{mL}$ spores of $F$. oxysporum f. sp. cucumerinum by dipping, cultured for one day, and then transferred to GTTs containing $10 \mathrm{~mL}$ of $1 / 8 \mathrm{MS}$ on the 6th day; T3, in which the cucumber seeds were only treated by dipping with $1 \times 10^{7} \mathrm{CFU} / \mathrm{mL}$ spores of T525, then transferred to plates with sterilized water and incubated at $28^{\circ} \mathrm{C}$ until the 6th day, after which they were transferred to GTTs containing $10 \mathrm{~mL}$ of $1 / 8 \mathrm{MS}$; T4, in which the cucumber seeds were only treated by dipping with $1 \times 10^{7}$ $\mathrm{CFU} / \mathrm{mL}$ spores of T525-F, then transferred to plates with sterilized water and incubated at $28^{\circ} \mathrm{C}$ until the 6th day, after which they were transferred to GTTs containing 10 $\mathrm{mL}$ of $1 / 8 \mathrm{MS}$; $\mathrm{T} 5$, in which the cucumber seeds were treated by dipping with $1 \times 10^{7} \mathrm{CFU} / \mathrm{mL}$ spores of $\mathrm{T} 525$, then transferred to plates with sterilized water and incubated at $28^{\circ} \mathrm{C}$. On the 4th day, the germinated seedlings were treated by dipping with $1 \times 10^{7} \mathrm{CFU} / \mathrm{mL}$ spores of $F$. oxysporum $\mathrm{f}$. sp. cucumerinum and cultured for one day until the 6th day, when they were transferred to GTTs containing $10 \mathrm{~mL}$ of $1 / 8 \mathrm{MS}$; T6, in which the cucumber seeds were treated by dipping with $1 \times 10^{7} \mathrm{CFU} / \mathrm{mL}$ spores of T525, then transferred to plates with sterilized water and incubated at $28^{\circ} \mathrm{C}$. On the 4th day, the germinated seedlings were treated by dipping with $1 \times 10^{7} \mathrm{CFU} / \mathrm{mL}$ spores of F. oxysporum f. sp. cucumerinum and cultured for one day, then transferred to GTTs containing $10 \mathrm{~mL}$ of $1 / 8 \mathrm{MS}$ on the 6 th day. On the 12th day, all treatments were observed. Every treatment was carried out in three repeat experiments with thirty replicates in total. For treatments $\mathrm{T} 1, \mathrm{~T} 3$, and T4, plant growth continued to be observed until the 24th day. The plates for all treatments were filled every day with sterilized water, and GTTs were filled to a constant volume of $10 \mathrm{~mL}$. Every five plants were photographed for every treatment.

\section{Results \\ Sequencing of the mycovirus genome}

From the 150 Trichoderma strains, we identified a strain designated HB40525 (or 525), containing typical dsRNAs of mycoviruses. Electrophoresis showed that the putative mycovirus fragment was approximately $3 \mathrm{~kb}$ in length (Fig. 1). Using next-generation sequencing (NGS) analysis, the contig-36 was determined to have a size of approximately $3 \mathrm{~kb}$ and to show high similarity to three mycoviruses: Alternaria longipes dsRNA virus 1 [25], Penicillium janczewskii Beauveria bassiana-like virus 1 [26], and Beauveria bassiana RNA virus 1 [27]. The strains exhibited sequence homology of $63.50 \%$ to Alternaria longipes dsRNA virus 1, YP_009052469.1 [25], $57.30 \%$ to Penicillium janczewskii Beauveria bassiana- 


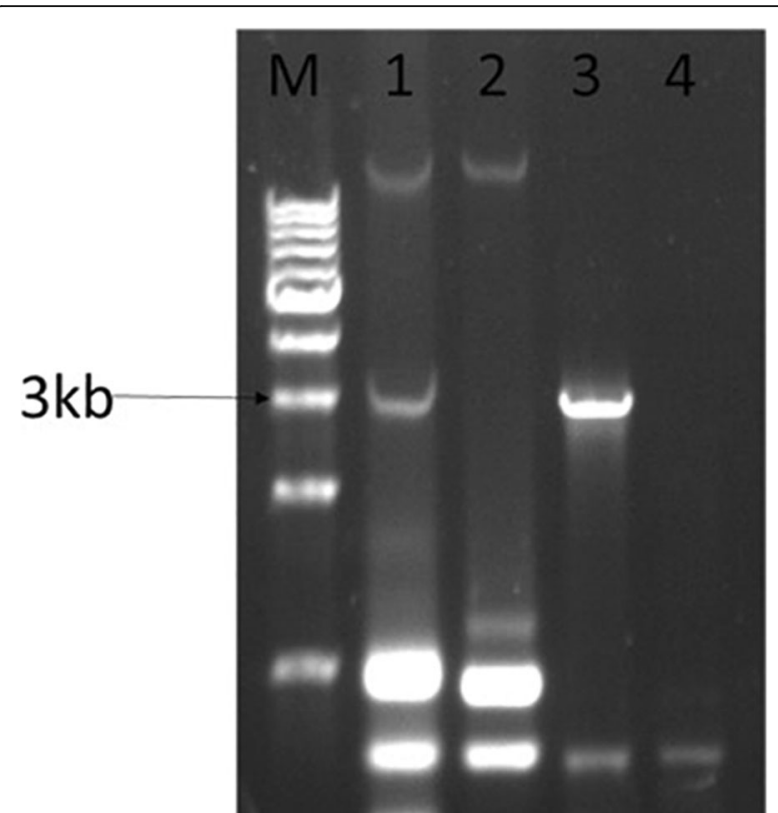

Fig. 1 Electrophoresis results. Lane M: DNA marker (10 kb ladder, TaKaRa); Line 1: dsRNA (approximately $3.2 \mathrm{~kb}$ ) extracted and purified from T. harzianum strain 525, subjected to electrophoresis in a $0.8 \%$ agarose gel and detected under a UV transilluminator. Lane 2: dsRNA from T. harzianum strain 525 after degradation by the enzymes of RNase I and DNase I and electrophoresis in a 0.8\% agarose gel, detected under a UV transilluminator. Lane 3: Detection of fragments of the dsRNA segments of mycovirus genomes by RT-PCR using specific primers

like virus 1, ALO50135.1 [26] and 57\% to Beauveria bassiana RNA virus 1, AKC57301.1 [27] (Additional file 1: Table S2). Using the sequence of contig-36, primers for RT-PCR were designed, and RT-PCR was performed. The CDNA sequences of the mycovirus without the $5^{\prime}$ and 3 ' termini were verified. The $5^{\prime}$ and $3^{\prime}$ terminal sequences of the cDNAs were obtained by the classic 5' RACE and 3' RACE cloning methods (Fig. 1, Additional file 1: Table S3 and Figure S1). The results indicated that the 3 ' terminus of the virus contained 15 poly(A) structures. Additional structures within the $5^{\prime}$ and $3^{\prime}$ termini were evaluated using RNA structure 3.0 (Additional file 1: Figure S2). The cDNA sequences and RACE sequences were assembled to obtain the complete sequence of the virus using
DNAMAN software. The resulting mycovirus genome was $3160 \mathrm{bp}$ (including the poly(A) tail) and was submitted to GenBank under accession number MH155602.

\section{Structure of the mycovirus genome}

In the genome sequence, the $\mathrm{G}+\mathrm{C}$ content was $51.98 \%$. The coding strand of the dsRNA exhibited two ORFs on the negative strand, which constituted the coding strand of the mycovirus. ORF-A (residues 1857-109) encoded an RNA-dependent RNA polymerase (RdRP), which was a protein of 582 amino acids with a molecular weight of approximately $66 \mathrm{kDa}$. ORF-B (residues 3047-1908) encoded a putative protein of 379 amino acids with a molecular weight of approximately $41 \mathrm{kDa}$. The positive strand harboured ORF C (residues 1076-1370), presumed to encode a hypothetical protein containing 94 amino acids with a poly(A) structure at the 3 ' terminus (Fig. 2, Additional file 1: Figure S2).

\section{Phylogenetic analysis of the mycovirus and its taxonomic status}

An NCBI BLASTN search identified five closely related fungal viruses with amino acid sequences similar to that of ORF-A: Alternaria longipes dsRNA virus 1 [25] (AlRV1, YP_009052469.1, homologous at 63\% sequence identity), Penicillium janczewskii Beauveria bassiana-like virus 1 [26] (PjBlV1, ALO50135.1, homologous at 57\% sequence identity); Beauveria bassiana RNA virus 1 [28] (BbNV-1, YP_009154711.1 homologous at 57\% sequence identity); Beauveria bassiana RNA virus 1 [27] (BbV1-A24, AKC57301.1, homologous at 57\% sequence identity); and Colletotrichum higginsianum non-segmented dsRNA virus 1 [29] (ChNRV1, YP_009177217.1, homologous at $55 \%$ sequence identity). The phylogenetic tree with the best model indicated that these fungal mycoviruses belonged to different families (Fusagraviridae, Megabirnaviridae, Totiviridae, Chrysoviridae), and the mycovirus of strain 525 belonged to the unclassified group structure [30] (Fig. 3a and Additional file 1: Table S4).

Based on the amino acid sequence of ORF-B, we found five closely related fungal viruses using NCBI BLASTn: AlRV1 (YP_009052468.1 homologous at 41\% sequence

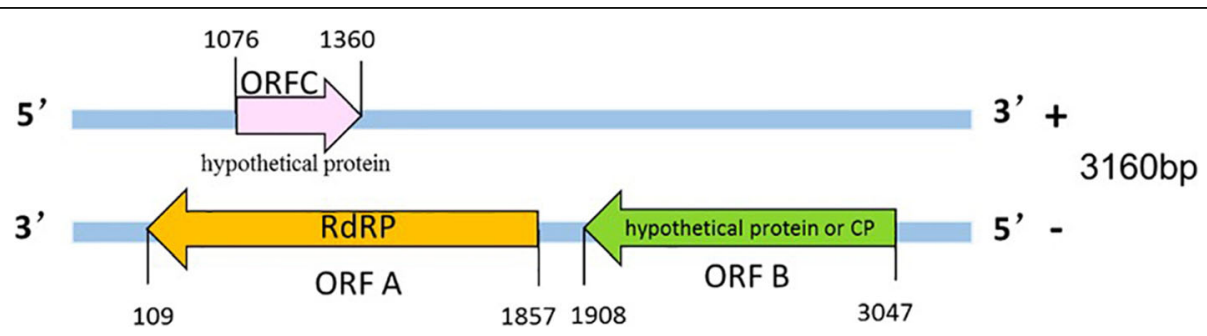

Fig. 2 Schematic representation of the genomic organization of ThMV1-525. The THRV1-525 genome is 3160 bp in length and contains two ORFs (ORF-A and ORF-B). ORF-A encodes the RNA-dependent RNA polymerase (RdRp); ORF-B encodes a hypothetical protein or coat protein (CP); the black lines indicate the 5'-UTR, 3'-UTR, and internal region 


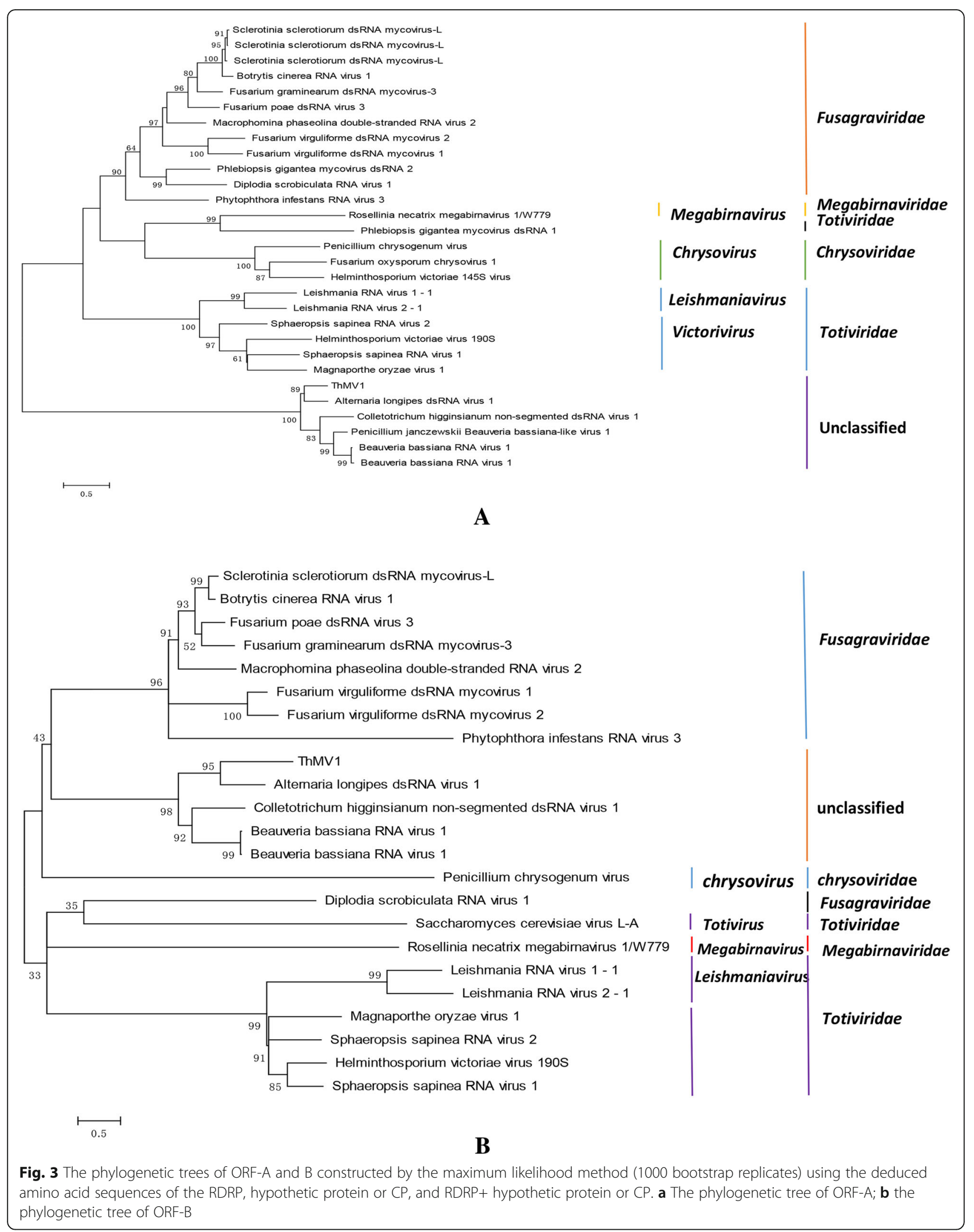


identity); BbNV-1 (YP_009154710.1 homologous at 42\% sequence identity); BbV1-A24 (AKC57300.1, homologous at 44\% sequence identity); PjBlV1 (ALO50134.1, homologous at $44 \%$ sequence identity); and ChNRV1 (YP_009177216.1, homologous at 43\% sequence identity). When all of these sequences were analysed using the best model, $\mathrm{LG}+\mathrm{G}+\mathrm{I}+\mathrm{F}$, ORF-B was grouped with the five mycoviruses with high similarity. The BbNV-1 protein is a putative coat protein, so ORF-B of strain 525 was considered to also encode a coat protein (Fig. $3 \mathrm{~b}$ and Additional file 1: Table S5).

From the BLAST results for ORF-C based on the amino acid sequences from the NCBI database, only one hypothetical protein from Mycolicibacterium conceptionense (WP_076212170.1) was discovered, with 34\% identity.

For the sake of completeness, the alignment that resulted from concatenating the aligned RdRP and coat protein sequences was also used to construct a single maximum likelihood phylogenetic tree (using the rtREV+G + I+F model) (Fig. 3c and Additional file 1: Table S6). The results were identical to the phylogenetic trees inferred using each protein independently. We concluded that the mycovirus from $T$. harzianum strain 525 was novel and unclassified, and we named it Trichoderma harzianum mycovirus 1 (ThMV1).

\section{Biological effects of ThMV1 on Trichoderma harzianum 525}

The elimination of the mycovirus from Trichoderma strain 525 was successfully performed using ribavirin and protoplasting/regeneration. RT-PCR of T525 and T525-F was performed to verify the elimination of ThMV1. The results showed that T525 exhibited a fragment length of $2997 \mathrm{bp}$, but T525-F did not contain the same fragment (Figs. 1 and 4a). No dsRNA from T525-F was detected by

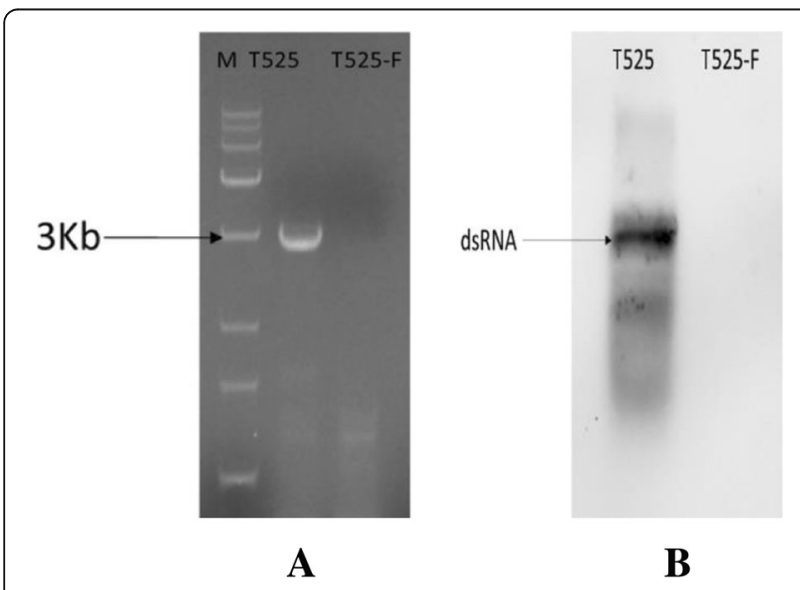

Fig. 4 Detection of T525- F by RT-PCR and Northern blotting. a Detection of T525 and strain T525-F (virus-free) by RT-PCR using specific primer pairs. b Northern blot detection of the dsRNAs of T525 and T525-F (virus-free) using a DIG probe
Northern blot analysis using a DIG-labelled probe, confirming that the mycovirus was successfully removed from T525-F (Fig. 4b).

When the growth rate of the two strains cultured on different media was measured, we found no statistically significant differences between them (Fig. $5 \mathrm{~d}$ and Additional file 1: Tables S7, S8, S9, and S10, ANOVA $P=0.095$ ), although on the 8th day, the hyphae of T525 had already covered the entire plate, while the hyphae of the T525$\mathrm{F}$ had not yet done so (Fig. $5 \mathrm{c}$ ). There were differences between the two growth media, however, with the hyphae growing almost twice as fast on PDA as on CZA (Fig. 5a and $\mathrm{b}$ and Additional file 1: Tables S7, S8, S9, and S10, ANOVA $P<0.001$ ). No significant strain-by-media interaction was observed (Fig. $5 \mathrm{~d}$ and Additional file 1: Table S9, ANOVA $P=0.389$ ), confirming that the similarity of growth rates between the two strains was independent of the medium. Because the CMD medium was thick and opaque, we could not measure the length of the hyphae accurately, but T525 grew more vigorously and densely than T525-F (Fig. 5b).

In contrast to the lack of difference between T525 and T525-F in terms of growth rates, the biomass produced by T525 was $13.25 \%$ greater than that of T525-F (Fig. 5e), a difference that was statistically significant (two-sample $t$ test: $t=2.117,38$ d.f., $P=0.041$ ) (Additional file 1: Tables S8 and S9). Thus, the presence of the mycovirus had a beneficial effect on the production of mycelia.

To evaluate biocontrol capabilities, we tested the antagonism of T525 and T525-F against three pathogenic fungi ( $F$. oxysporum f.sp. cucumebrium Owen, B. cinerea and $F$. oxysporum $\mathrm{f}$. sp. vasinfectum). There were no obvious differences in vitro (Additional file 1: Figure S2), but the control effectiveness towards cucumber wilt disease caused by F. oxysporum f.sp. cucumebrium Owen was significantly different in vivo. While the cucumber seedlings in the T1, T3, and T4 treatments all grew well, little difference existed in the observed growth trend; all of the cucumber seedlings of T3 grew more vigorously and exhibited more fibrous roots than $\mathrm{T} 1$ and grew higher than T4 by the 12th day (Fig. 6). Moreover, on the 24th day, the cucumber seedlings of T3 were all larger than those of $\mathrm{T} 1$ and $\mathrm{T} 4$, with larger true leaves and more fibrous roots (Fig. 7), which indicated that $\mathrm{T} 525$ had the potential to function to improve cucumber growth. The T2 seedlings infected only with $F$. oxysporum f. sp. cucumebrium Owen were all withered 7 days after inoculation, which indicated that the cucumber species exhibited no resistance to this pathogen. However, the growth trends of T6 were all better than those of T2 and T5. T2 showed severe disease symptoms, whereas the growth trend of $\mathrm{T} 5$ only showed the initial stage of withering, with all leaves beginning to turn yellow (Fig. 6 and Additional file 1: Figure S3). 


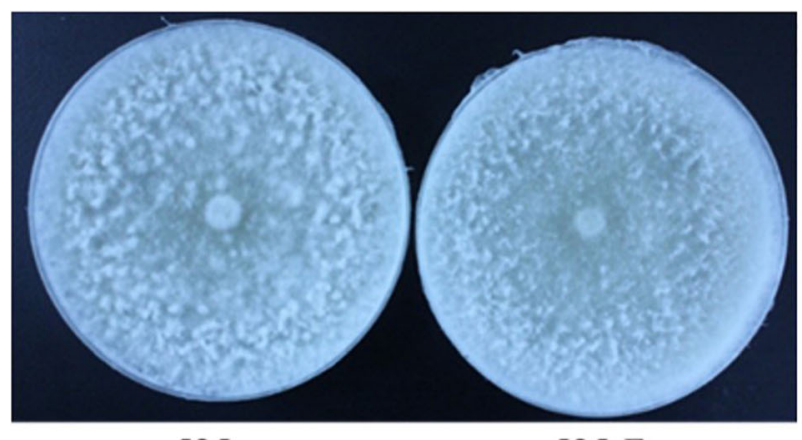

525

A
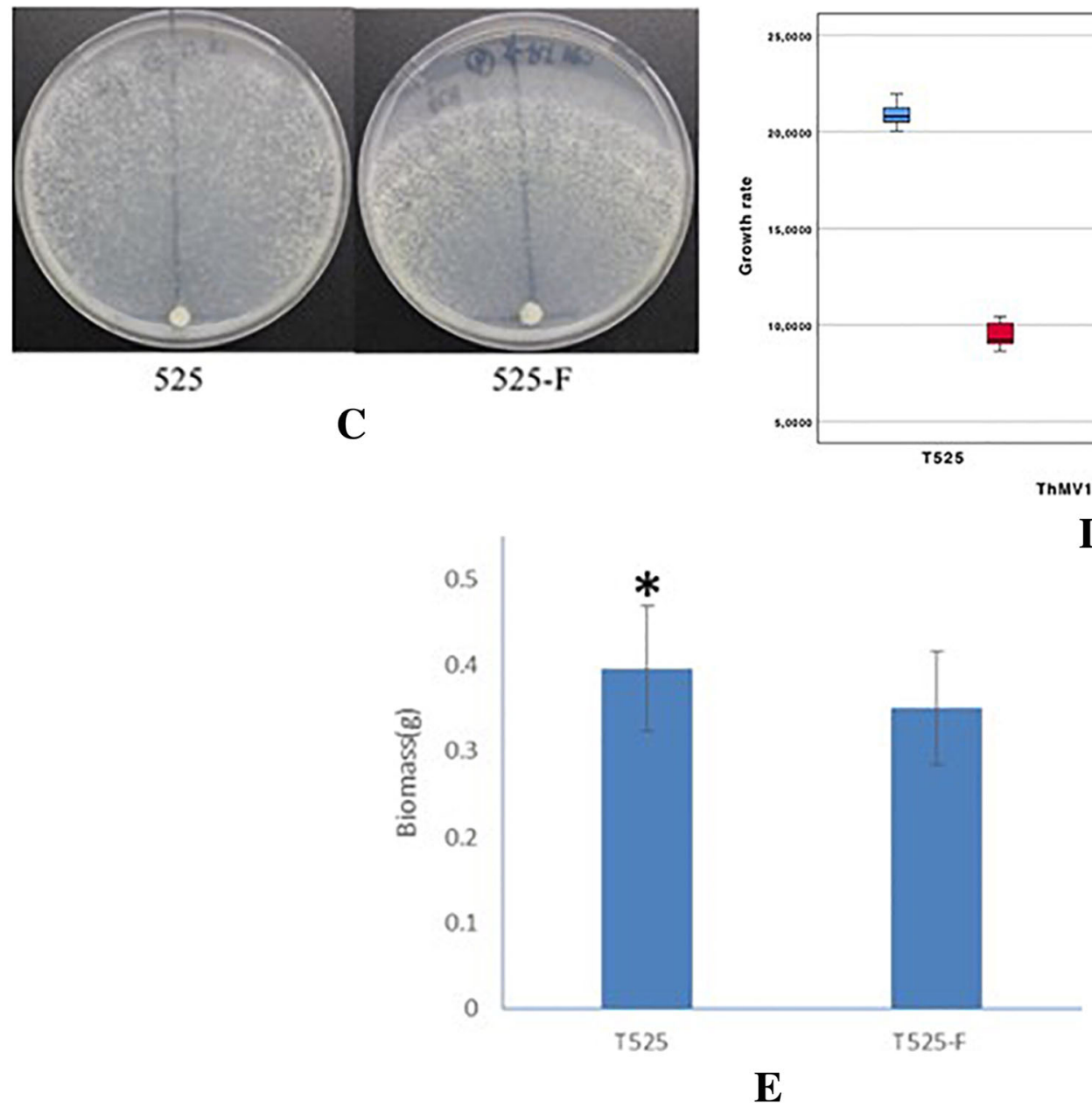

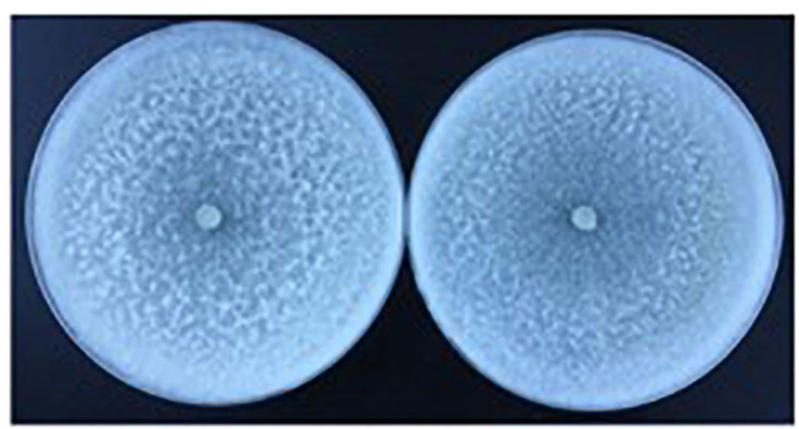

525

B

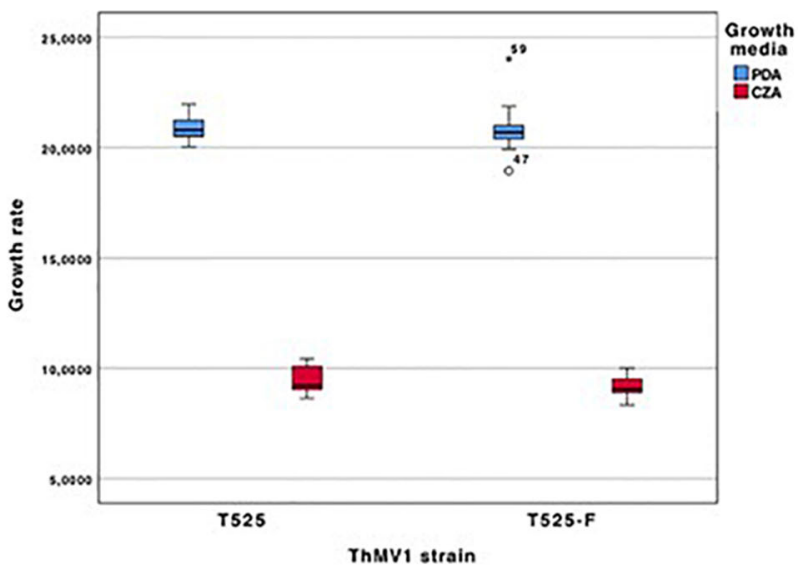

D

Fig. 5 Colony morphology of T525 and T525-F after 7 days of culture on PDA (potato dextrose agar medium), CMD (corn meal dextrose agar medium), and CZA (Czapek-Dox agar medium). a PDA medium. b CMD medium. c CZA medium. d Growth rate of strain 525 and strain 525-F after 3 days on PDA and CZA medium, respectively. E: Biomass comparison of T525 and T525-F. The bars (D-F) represent the standard deviation from the mean (d: $n=30 ; \mathbf{e : ~} n=20)$. "**" indicates that differences are statistically significant $(p<0.05)$

These results together indicate that $\mathrm{T} 525$ possesses the capacity to promote plant growth, while T525-F could improve the pathogen resistance of plants. The milder disease symptoms of cucumber seedlings treated with T525-F implied that ThMV1 interacted with its host,
T525, and decreased the host's biocontrol efficiency against plant disease. Plants treated with T525 (T3) appeared to grow significantly better than those that did not receive this treatment (T1) or were treated with T525-F (T4), and T1 was observed to promote better growth than T4. 


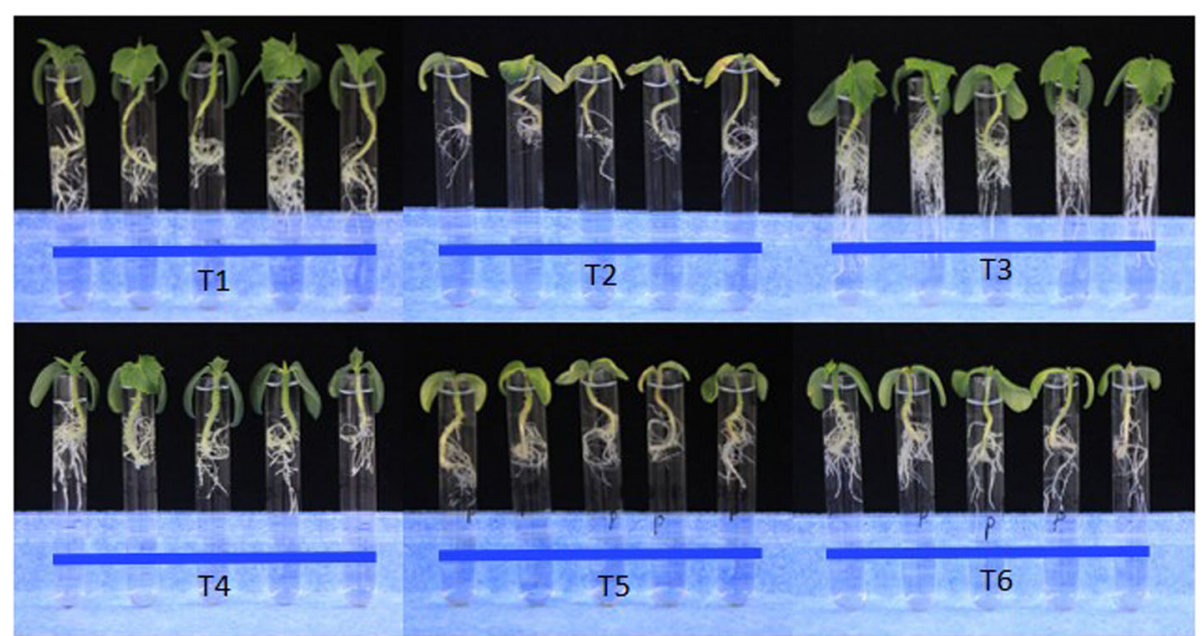

Fig. 6 Evaluation of the biocontrol effects of T525 and T525-F. T1: healthy cucumber without any treatment; T2: cucumber seeds treated only with F. oxysporum f. sp. Cucumerinum; T3: cucumber seeds treated only with Trichoderma strain 525; T4: cucumber seeds treated only with Trichoderma strain 525-F. T5: cucumber plants treated only with Trichoderma strain 525 and F. oxysporum f. sp. Cucumerinum; T6: cucumber plants treated only with Trichoderma strain 525-F and F. oxysporum f. sp. Cucumerinum

\section{Discussion}

This study describes a new mycovirus found in T. harzianum strain 525, which was isolated from grassland soils in Inner Mongolia, China. Through sequence alignment, the mycovirus was found to exhibit high sequence homology with Alternaria longipes dsRNA virus 1, Penicillium janczewskii Beauveria bassiana-like virus 1, Beauveria bassiana RNA virus 1, Beauveria bassiana RNA virus 1 , and Colletotrichum higginsianum non-segmented dsRNA virus 1 , with sequence identities of 44-50.5\% (Additional file 1: Table S6). Based on phylogenetic analysis of its amino acid sequence, this mycovirus was identified as a new unclassified type of mycovirus.

To date, only three isolates of mycoviruses have been recovered from Trichoderma spp. Jom-in and Akarapisan (2009) isolated two mycoviruses with sizes of $0.7 \mathrm{~kb}$ and $1.1 \mathrm{~kb}$ from Trichoderma spp., although their taxonomic status was not analysed or classified [31]. Yun et al. (2016) noted that mycoviruses of Lentinula edodes are widespread in fungi [20]. They isolated 32 strains containing dsRNA from 315 Trichoderma strains, which were divided into 15 groups according to dsRNA electrophoresis patterns. Although colony morphological characteristics differed among the groups [20], accurate mycovirus sequences were not obtained. Lee et al. (2017) also isolated an unclassified mycovirus from $T$. atroviride referred to as Trichoderma atroviride mycovirus 1 (TaMV1) [30].

Previous studies on the mycoviruses of fungal pathogens have shown that they can affect the virulence of host fungi. For instance, Li et al. (2015) found that Fusarium graminearum Hypovirus 2 (FgHV2/JS16) could significantly reduce the pathogenicity of Fusarium graminearum by inhibiting the production of deoxynivalenol (DON) toxin [32]. Zhang et al. (2014) found that the dsRNA mycovirus Rhizoctonia solani RNA virus 2 (RsRV2) from the rice sheath blight isolate D122 strain, which belongs to the family Paritiviridae, increased the virulence of host fungus towards plants [11]. Jom-in et al. (2009) tried to remove the mycovirus from TM10 and TM20 hosts but was unable to obtain Trichoderma strain-free mycovirus [31]. Jom-in investigated the antagonistic activity of Trichoderma spp. without mycoviruses and indirectly verified

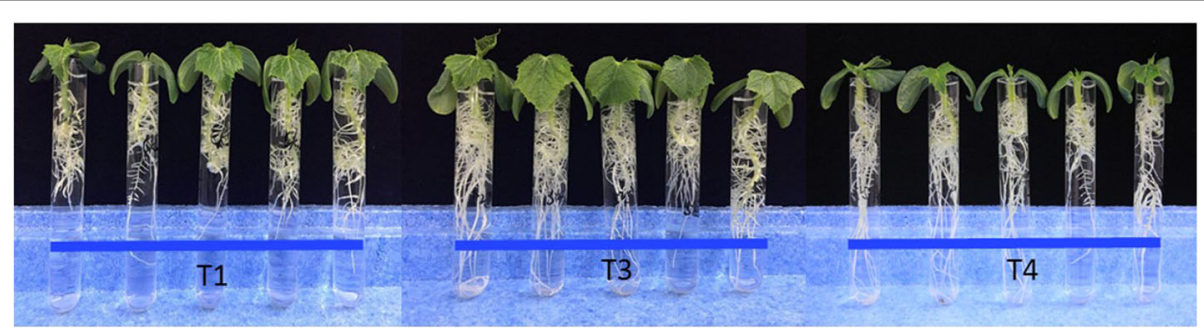

Fig. 7 Evaluation of the difference in the improvement of cucumber growth by T525 and T525-F. T1: healthy cucumber without any treatment after 24 d; T3: cucumber seeds treated only with Trichoderma strain 525 after 24 d; T4: cucumber seeds treated only with Trichoderma strain 525-F after 24 d 
that TM10 and TM20 mycoviruses decreased the antagonistic action of Trichoderma spp. [31]. In our study, the mycovirus of T525 was successfully eliminated, and ThMV1 did not affect the antagonistic activities of the host in vitro, although it obviously decreased the biocontrol efficiency of T525 against $F$. oxysporum f.sp. cucumebrium Owen in vivo. ThMV1 also exhibited the potential to induce plant growth. This study is the first to report the biocontrol traits of a mycovirus from Trichoderma spp., highlighting the interactions between $T$. harzianum, $F$. oxysporum f. sp. cucumebrium Owen and ThMV1.

\section{Conclusions}

ThMV1 is the first reported mycovirus that not only affects the biomass of the host strain but also reduces the biocontrol function of Trichoderma and promotes the growth of plants. This work provides insight into how to locate and isolate mycoviruses that could potentially affect the biocontrol function of Trichoderma. The interaction mechanisms among the mycovirus, Trichoderma, pathogen, and plant will be the focus of future investigations.

\section{Additional file}

Additional file 1: Table S1. Information on Trichoderma strains collected from Xinjiang, Inner Mongolia, Jilin and Heilongjiang Provinces of China. Table S2. High identities between contig 36 and other mycoviruses as determined by next-generation sequencing (HGS). Table S3. The primers used for 5' RACE and 3' RACE of the mycovirus genome sequence. Table S4. RdRP data of mycoviruses used in the phylogenetic analysis, including the identities of RdRP between ThMV1 and the compared mycoviruses. Table S5. CP data of mycoviruses used in the phylogentic analysis, including the CP identities between ThMV1 and the compared mycoviruses. Table S6. RdRP+CP data of mycoviruses used in the phylogenetic analysis, including the identities of RdRP+CP between ThMV1 and the compared mycoviruses. Table S7. Statistical analysis of hyphae from T525 and T525-F after 5 days on PDA medium by the Mann-Whitney $U$ test. Table S8. Average growth rate, standard deviation, and statistical analysis of hyphae from T525 and T525-F after 8 days on CZA medium by the Mann-Whitney $U$ test. Table S9. Biomass comparison, standard deviation, and statistical analysis of biomass between T525 and T525-F by the Mann-Whitney U test. Table S10. Statistical analysis for the comparison of biomass between T525 and T525-F by using SPSS. Figure S1. Electrophoresis images of 5' RACE and 3' RACE results for the genome sequence of the T525 mycovirus. Figure S2. The secondary RNA structures of the $5^{\prime} U T R$ and $3^{\prime} U T R$. Figure S3. The antagonistic characteristics of T525 and T525-F against F. oxysporum f.sp. cucumebrium Owen, B. cinerea and F. oxysporum f. sp. vasinfectum. Figure S4. Experimental flow chart for evaluating the biocontrol capabilities of $F$. oxysporum f. sp. cucumebrium Owen in cucumber. (DOCX 293 kb)

\section{Abbreviations}

$\%$ : Percentage; ${ }^{\circ} \mathrm{C}$ : Degrees Celsius; ANOVA: Analysis of variance; BLAST: Basic Local Alignment Search Tool; bp: Base pair; CDNA: Complementary DNA; CK: Control check; CMD: Corn Meal Dextrose Agar; CP: Coat protein; CZA: Czapek-Dox; DIG: Digoxigenin; dsDNA: Double-strand DNA; dsRNA: Double-stranded RNA; et al.: And others; Fig: Figure; GTT: Glass test tube; i.e.: That is; KB: Kilobase; MEGA: Molecular evolutionary genetics analysis; ml: Millilitre; mm: Millimetre; MS: Murashige and Skoog; NCBI: National Center for Biotechnology Information; NGS: Next-generation sequencing; NO.: Number; ORF: Open reading frame; $P$ : $P$ value; PCR: Polymerase chain reaction; PDA: Potato dextrose agar; pmol: Parts per million; RACE: Rapid amplification of cDNA ends; RdRP: RNA-dependent RNA polymerase; Rpm: Revolutions per minute; rRNA: Ribosomal RNA (rRNA); RTPCR: Reverse-transcription polymerase chain reaction; ssDNA: Single-strand DNA; ssRNA: Single-strand RNA; T525: The 525 strain with mycovirus; T525F: The 525-F strain without mycovirus; TDT: Terminal deoxynucleotidyl transferase; UV: Ultraviolet; $\mu \mathrm{l}:$ Microliter; $\mu \mathrm{M}: 1 \mathrm{pmol} / \mu \mathrm{L}$

\section{Acknowledgements}

We are grateful to Prof. Santiago F. Elena (I²SysBio-CSIC, Valencia, Spain) for many helpful suggestions during this research.

\section{Authors' contributions}

$\mathrm{CL}$ and ML performed the experiments; ETR, JM and JZ isolated Trichoderma spp., CL and BW analysed the data and wrote the manuscript; BW and XJ designed the study. All authors read and approved the final manuscript.

\section{Funding}

This work was supported by the National Key Research and Development Plan (Chemical Fertilizer and Pesticide Reducing Efficiency Synergistic Technology Research and Development): Science and Technology Foundation of the Ministry of Science and Technology of China (2014FY120900-4) granted to Xiliang Jiang; Natural Science Foundation of Beijing, China: Exploration of Mycovirus of Trichoderma and Their Effects on the Host Biology (No. 6192022) granted to Beilei Wu; Demonstration of Comprehensive Prevention and Control Technology of Non-point Source Pollution in Main Vegetable-Producing Areas of Huang Huai Hai

(SQ2018YFD080026) granted to Beilei Wu.

Ethics approval and consent to participate

This article does not contain any studies with human participants performed by any of the authors.

Consent for publication

Informed consent was obtained from all participants included in the study.

Competing interests

The authors declare that they have no competing interests.

Received: 1 December 2018 Accepted: 5 August 2019

Published online: 11 September 2019

References

1. Hollings M. Viruses associated with a die-back disease of cultivated mushroom. Nature. 1962;4858:962-5.

2. Buck KW. Fungal virology-an overview. Boca Raton Florida: CRC Press, Boca Raton; 1986. p. 1-84

3. Nibert ML, Ghabrial SA, Maiss E, Lesker T, Vainio EJ, Jiang D, Suzuki N. Taxonomic reorganization of family Partitiviridae and other recent progress in partitivirus research. Virus Res. 2014;188:128-41.

4. Kotta-Loizou I, Coutts RH. Mycoviruses in Aspergilli: a comprehensive review. Front Microbiol. 2017:8:1699.

5. Ghabrial SA, Castón JR, Jiang D, Nibert ML, Suzuki N. 50-plus years of fungal viruses. Virology. 2015;479(60):356-68.

6. Ghabrial SA, Suzuki N. Viruses of plant pathogenic fungi. Annu Rev Phytopathol. 2009;47:353-84.

7. Ikeda K, Nakamura H, Matsumoto N. Mycelial incompatibility operative in pairings between single basidiospore isolates of Helicobasidium mompa. Mycol Res. 2003;107(7):847-53.

8. Varga J, Toth B, Vagvolgyi C. Recent advances in mycovirus research. Acta Microbiol Immunol Hung. 2003;50:77-94.

9. Pandey B, Naidu RA, Grove GG. Detection and analysis of mycovirus related RNA viruses from grape powdery mildew fungus Erysiphe necator. Arch Virol. 2018;163:1019-30.

10. Chu YM, Jeon JJ, Yea SJ, Kim YH, Yun SH, Lee YW, Kim KH. Double-stranded RNA mycovirus from Fusarium graminearum. Appl Environ Microbiol. 2002; 68:2529-34.

11. Guo ZJ, Wu YH. Proceedings of the annual meeting of Chinese Society for Plant Pathology, LiaoNing; 2014.

12. Nuss DL. Hypovirulence: mycoviruses at the fungal-plant interface. Nat Rev Microbiol. 2005;3(8):632-42.

13. Chiba S, Salaipeth L, Lin YH, Sasaki A, Kanematsu S, Suzuki N. A novel bipartite double-stranded RNA mycovirus from the white root rot fungus Rosellinia 
necatrix: molecular and bio- logical characterization, taxonomic considerations, and potential for biological control. J Virol. 2009;83:12801-12.

14. Xie J, Jiang D. New insights into mycoviruses and exploration for the biological control of crop fungal diseases. Annu Rev Phytopathol. 2014;52: 45-68.

15. Ikeda K, Inoue K, Kida C, Uwamori T, Sasaki A, Kanematsu S, Park P. Potentiation of mycovirus transmission by zinccompounds via attenuation of heterogenic incompatibility in Rosellinia necatrix. Appl Environ Microbiol. 2013;79(12):3684-91.

16. Milgroom MG, Cortesi P. Biological control of chestnut blight with hypovirulence: a critical analysis. Annu Rev Phytopathol. 2004;42:311-38.

17. Schmitt MJ, Breinig F. Yeast viral killer toxins: lethality and self-protection. Nat Rev Microbiol. 2006;4(3):212-21.

18. Grente J, Berthelay-Sauret S. Biological control of chestnut blight. In: MacDonald WL, Cech FC, Luchok J, Smith HC, editors. Proceedings of the American chestnut symposium. Morgantown: West Virginia University Press; 1978. p. 30-4.

19. Howell CR. Mechanisms employed by Trichoderma species in the biological control of plant diseases: the history and evolutions of current concepts. Plant Dis. 2003;87:4-10.

20. Yun SH, Song HL, So KK, Kim JM, Kim DH. Incidence of diverse dsRNA mycoviruses in Trichoderma spp. causing green mold disease of shiitake Lentinula edodes. FEMS Microbiol Lett. 2016;363(19):fnw220.

21. DePaulo J, Powell C. Extraction of double-stranded RNA from plant tissues without the use of organic solvents. Plant Dis. 1995;79(3):246-8.

22. Potgieter AC, Page NA, Liebenberg J, Wright IM, Landt O, van Dijk AA. Improved strategies for sequence-independent amplification and sequencing of viral dsRNA genomes. J Gen Viro. 2009;90:1423-32.

23. Hou Z, Xue C, Peng Y, Katan T, Kistler HC, Xu JR. A mitogen-activatedprotein kinase gene (MGV1) in Fusarium graminearum is required for femalefertility, heterokaryon formation, andplant infection. Mol Plant Microbe IN. 2002;15: $1119-27$.

24. Herrero N, Zabalgogeazcoa I. Mycoviruses infecting the endophytic and entomopathogenic fungus Tolypocladium cylindrosporum. Virus Res Sep. 2011;160(1-2):409-13.

25. Lin Y, Zhang H, Zhao C, Liu S, Guo L. The complete genome sequence of a novel mycovirus from Alternaria longipes strain HN28. Arch Virol. 2015;160(2): $577-80$

26. Nerva L, Ciuffo M, Vallino M, Margaria P, Varese GC, Gnavi G, Turina M. Multiple approaches for the detection and characterization of viral and plasmid symbionts from a collection of marine fungi. Virus Res. 2015;219: 22-38.

27. Koloniuk I, Hrabáková L, Petrzik K. Molecular characterization of a novel amalgavirus from the entomopathogenic fungus Beauveria bassiana. Arch Virol. 2015;160(6):1585-8.

28. Kotta-Loizou I, Sipkova J, Coutts RH. Identification and sequence determination of a novel double-stranded RNA mycovirus from the entomopathogenic fungus Beauveria bassiana. Arch Virol. 2015;160(3):873-5.

29. Campo S, Gilbert KB, Carrington JC. Small RNA-based antiviral defense in the Phytopathogenic fungus Colletotrichum higginsianum. PLoS Pathog. 2016;12(6):e1005640

30. Lee SH, Yun SH, Chun J, Kim DH. Characterization of a novel dsRNA mycovirus of Trichoderma atroviride NFCF028. Arch Virol. 2017;162(4):1073-7.

31. Jom-in, S., Akarapisan, A. Characterization of double-stranded RNA in Trichoderma spp. isolates in Chiang Mai province. J Agr Tech. 2009:5(2):261-70

32. Li P, Zhang H, Chen X, Qiu D, Guo L. Molecular characterization of a novel hypovirus from the plant pathogenic fungus Fusarium graminearum. Virology. 2015;481:151-60.

\section{Publisher's Note}

Springer Nature remains neutral with regard to jurisdictional claims in published maps and institutional affiliations.

Ready to submit your research? Choose BMC and benefit from:

- fast, convenient online submission

- thorough peer review by experienced researchers in your field

- rapid publication on acceptance

- support for research data, including large and complex data types

- gold Open Access which fosters wider collaboration and increased citations

- maximum visibility for your research: over $100 \mathrm{M}$ website views per year

At $\mathrm{BMC}$, research is always in progress.

Learn more biomedcentral.com/submissions 